\title{
La centralità della volontà del paziente incapace in una recente sentenza della Corte di Cassazione
}

\author{
Paola Delbon ', Adelaide Conti ${ }^{1}$ \\ Cattedra di Medicina Legale. Centro di Studio e di Ricerca di Bioetica dell'Università degli Studi di Brescia \\ (In coll. con l'IRCCS Centro San Giovanni di Dio Fatebenefratelli)
}

\begin{abstract}
A recent judgement by the Court of Cassation about a request - by the legal guardian of an incompetent patient in permanent vegetative state - of suspension of artificial nutrition and hydration, gives important instructions about informed consent, right of self-determination, and right to refuse treatment in competent and incompetent patients, with particular regard to the role of legal guardian and to the validity of advance care directives.
\end{abstract}

Keywords: informed consent, incompetent patient, legal guardian, advance care directives

The importance of the will of incompetent patient in a recent judgement by the Court of Cassation. Pratica Medica \& Aspetti Legali 2008; 2(3): 143-147

\section{INTRODUZIONE}

Il consenso informato ai trattamenti sanitari costituisce ormai un elemento imprescindibile nel rapporto medico-paziente, presupposto indispensabile per l'esecuzione di qualsiasi trattamento ed espressione del principio di autonomia, e specificamente del diritto all'autodeterminazione, ovvero del diritto di ciascun paziente di assumere decisioni libere e consapevoli in relazione al proprio stato di salute.

Una recente sentenza della Corte di Cassazione (Corte di Cassazione, sentenza n. 21748 del 16 ottobre 2007) è intervenuta in una lunga vicenda giudiziaria scaturita dalla richiesta di sospensione dell'alimentazione e dell'idratazione artificiali di una persona in stato vegetativo permanente dal 1992, avanzata dal tutore della stessa, affrontando proprio la tematica del consenso ai trattamenti e del parallelo diritto di rifiutare le cure, in relazione sia al soggetto capace sia al soggetto incapace di formare ed esprimere la propria volontà in materia.

Anzitutto la Corte avverte l'esigenza di precisare lo stato dell'ordinamento giuridico italiano rispet- to al recepimento della cosiddetta "Convenzione europea sui diritti umani e la biomedicina” approvata dal Consiglio d'Europa a Strasburgo nel 1996 e aperta alla firma degli Stati membri a Oviedo nel 1997, contenente importanti norme in materia di autonomia, informazione, consenso, tutela dei pazienti incapaci: «sebbene il Parlamento ne abbia autorizzato la ratifica con la legge 28 marzo 2001, n. 145 , la Convenzione di Oviedo non è stata a tutt'oggi ratificata dallo Stato italiano. Ma da ciò non consegue che la Convenzione sia priva di alcun effetto nel nostro ordinamento. Difatti, all'accordo valido sul piano internazionale, ma non ancora eseguito all'interno dello Stato, può assegnarsi - tanto più dopo la legge parlamentare di autorizzazione alla ratifica - una funzione ausiliaria sul piano interpretativo: esso dovrà cedere di fronte a norme interne contrarie, ma può e deve essere utilizzato nell'interpretazione di norme interne al fine di dare a queste una lettura il più possibile ad esso conforme».

In primo luogo la Corte sottolinea che «il consenso informato ha come correlato la facoltà non solo di scegliere tra le diverse possibilità di trattamento medico, ma anche di eventualmente rifiutare la terapia e di decidere consapevolmente di interrom- 
perla, in tutte le fasi della vita, anche in quella terminale».

Anche il Comitato Nazionale per la Bioetica (CNB) [1] ha affermato espressamente la libertà di autodeterminazione del paziente: «ferma l'esistenza del diritto alla salute e una certa nota di doverosità nel conservarla, ricavabile dall'insieme delle disposizioni costituzionali, sussisterebbe tuttavia la facoltà di sacrificare tale diritto o più precisamente i beni oggetti del diritto».

D'altra parte, la valorizzazione dell'autonomia del soggetto in relazione a questa fase complessa e delicata della malattia e della vita si impone in modo particolare in virtù della dimensione fortemente soggettiva e non puramente tecnica delle decisioni che caratterizzano tale fase dell'esistenza.

Tale approccio combina pertanto componenti soggettive e oggettive: la decisione in merito ai trattamenti non è dunque unilaterale, ma esige anche la conoscenza del "punto di vista" proprio del paziente.

Ciò appare coerente anche con la definizione di salute elaborata dall'Organizzazione Mondiale della Sanità (Costituzione dell'Organizzazione Mondiale della Sanità, firmata a New York il 22 luglio 1946, approvata dall'Assemblea Federale il 19 dicembre 1946, ed entrata in vigore il 7 aprile 1948), secondo la quale la salute è «uno stato di completo benessere fisico, mentale e sociale, e non consiste solo in un'assenza di malattia o d'infermità».

La salute dunque «non viene più intesa esclusivamente in termini oggettivi, come assenza di malattia, ma deve essere considerata in relazione alla percezione che il soggetto ha di sé, del proprio stato fisico e mentale» [2]: diviene pertanto fondamentale il riferimento all'esperienza di vita del paziente, ovvero al suo sistema valoriale di riferimento, a livello culturale, religioso e familiare. Così anche la Corte di Cassazione rivendica il riferimento primario alla volontà dell'interessato coerentemente "con la nuova dimensione che ha assunto la salute, non più intesa come semplice assenza di malattia, ma come stato di completo benessere fisico e psichico, e quindi coinvolgente, in relazione alla percezione che ciascuno ha di sé, anche gli aspetti interiori della vita come avvertiti e vissuti dal soggetto nella sua esperienza».

\section{LA VOLONTÀ NEL PAZIENTE INCAPACE}

Le difficoltà sorgono evidentemente nelle ipotesi in cui il paziente non sia in grado di formare e/o esprimere la propria volontà ai curanti, e in par- ticolare "non abbia, prima di cadere in tale condizione, allorché era in pieno possesso delle sue facoltà mentali, specificamente indicato, attraverso dichiarazioni di volontà anticipate, quali terapie egli avrebbe desiderato ricevere e quali invece avrebbe inteso rifiutare nel caso in cui fosse venuto a trovarsi in uno stato di incoscienza».

Nell'ipotesi in cui il soggetto sia legalmente incapace, ovvero in presenza di un legale rappresentante del paziente stesso, la legittimazione ad esprimere il consenso ai trattamenti sanitari spetta al tutore, in qualità di titolare del poteredovere di cura dell'incapace.

La funzione di cura del tutore e la sua titolarità delle decisioni inerenti la cura dell'incapace contribuiscono peraltro alla realizzazione del principio ispiratore delle norme sugli incapaci, ovvero "quello di superare le diversità attraverso l'integrazione della capacità» [3], consentendo ai soggetti, altrimenti incapaci, di realizzare comunque le posizioni soggettive delle quali sono titolari in condizione di parità con gli altri soggetti (capaci) dell'ordinamento giuridico, e dunque di recuperare una situazione di effettiva eguaglianza rispetto agli stessi: "consentire all'incapace di esprimersi rispetto al consenso informato attraverso il proprio tutore e quindi rifiutare i trattamenti sanitari offensivi della sua dignità, colma una lacuna, riduce una diversità» [4].

Per quanto concerne gli atti rispetto ai quali si estrinseca il potere-dovere di cura della persona, e dunque le scelte relative ai trattamenti a tutela della salute, i genitori o il tutore dell'interdetto/a «esprimono all'esterno il consenso non in quanto investiti di una funzione sostitutiva di rappresentanza, bensì esercitando un ruolo di natura personale che impone loro di tener conto della personalità del minore o dell'interdetto [...], e conseguentemente di decidere "con lui" e non "per lui"» [5]. Naturalmente, come sottolinea la Corte, il poteredovere di cura del tutore deve estrinsecarsi nell'interesse dell'incapace e dunque il suo esercizio non può essere indiscriminato: "Assodato che i doveri di cura della persona in capo al tutore si sostanziano nel prestare il consenso informato al trattamento medico avente come destinatario la persona in stato di incapacità, si tratta di stabilire i limiti dell'intervento del rappresentante legale».

In particolare, la Corte precisa che «la rappresentanza del tutore è sottoposta a un duplice ordine di vincoli: egli deve, innanzitutto, agire nell'esclusivo interesse dell'incapace; e, nella ricerca del best interest, deve decidere non "al posto" dell'incapace, né "per" l'incapace, ma "con" l'incapace: quindi, ricostruendo la presunta volontà del paziente incosciente, già adulto prima di cadere in tale stato, tenendo conto dei desideri da lui espressi prima della perdita della coscienza, ovvero inferendo quella volontà dalla sua personalità, dal suo stile di vita, dalle sue inclinazioni, dai suoi 
valori di riferimento e dalle sue convinzioni etiche, religiose, culturali e filosofiche».

Il primo "limite" individuato dalla Corte è rappresentato dall' "esclusivo interesse dell'incapace", un limite che trova giustificazione nella stessa natura intrinseca dell'istituto della "tutela".

Tale principio è peraltro affermato anche nell'art. 6 ("Protezione delle persone che non hanno la capacità di dare il consenso") della citata "Convenzione sui diritti dell'uomo e la biomedicina”, che condiziona esplicitamente la possibilità di effettuare un intervento nei confronti di una persona priva della capacità di dare il consenso, alla realizzazione di un diretto beneficio per l'incapace.

La centralità dell'interesse del paziente emerge dunque chiaramente dalla norma citata, e specificamente dal riferimento al "diretto beneficio" del paziente incapace quale requisito necessario per l'effettuazione di un trattamento nei suoi confronti.

Il nostro ordinamento giuridico ha esplicitato tale requisito anche nell'ambito della disciplina della sperimentazione clinica: l'art. 5 ("Sperimentazione clinica su adulti incapaci di dare validamente il proprio consenso informato") del D. Lgs.vo 24 giugno 2003, n. 211 ("Attuazione della direttiva 2001/20/CE relativa all'applicazione della buona pratica clinica nell'esecuzione delle sperimentazioni cliniche di medicinali per uso clinico") subordina la partecipazione ad uno studio clinico di soggetti adulti incapaci alla condizione, tra le altre, che «a) sia stato ottenuto il consenso informato del rappresentante legale; il consenso deve rappresentare la presunta volontà del soggetto e può essere ritirato in qualsiasi momento senza pregiudizio per il soggetto stesso".

Tale limite non può essere peraltro completamente svincolato dal secondo limite indicato dalla Corte proprio per la natura non esclusivamente oggettiva della determinazione di ciò che corrisponde al miglior interesse del paziente: la Corte afferma infatti che il tutore "nella ricerca del best interest, deve decidere non "al posto" dell'incapace né "per" l'incapace, ma "con" l'incapace», ovvero attraverso la ricostruzione della presunta volontà del soggetto incapace.

A tal fine la Cassazione fa espressamente riferimento alla manifestazione da parte del paziente della propria volontà, prima della perdita della coscienza, quale elemento da tenere in considerazione per la ricostruzione della sua volontà, conformemente a quanto stabilito dalla citata Convenzione sui diritti dell'uomo e la biomedicina, che all'art. 9 (“Desideri precedentemente espressi”) stabilisce che «i desideri precedentemente espressi a proposito di un intervento medico da parte di un paziente che, al momento dell'intervento, non è in grado di esprimere la sua volontà saranno tenuti in considerazione».
La Corte di Cassazione individua dunque una ipotesi privilegiata per la determinazione della volontà del paziente: se i desideri espressi in precedenza contemplati all'art. 9 della Convenzione non hanno lo stato giuridico di documenti vincolanti per i curanti, essi, laddove trasmessi dal paziente, possono costituire un elemento fondamentale sulla base del quale il tutore può dare contenuto alla decisione che è legittimato ad esprimere, nell'interesse dell'incapace.

Tali documenti possono peraltro costituire un importante strumento di supporto nel processo decisionale anche per il personale sanitario, secondo quanto stabilito dal Codice di deontologia medica, la cui ultima versione è stata approvata dalla Federazione Nazionale degli Ordini dei Medici Chirurghi e degli Odontoiatri in data 16 dicembre 2006. Gli artt. 35 ("Acquisizione del consenso") e 38 ("Autonomia del cittadino e direttive anticipate") stabiliscono al riguardo rispettivamente che «il medico deve intervenire, in scienza e coscienza, nei confronti del paziente incapace, nel rispetto della dignità della persona e della qualità della vita, evitando ogni accanimento terapeutico, tenendo conto delle precedenti volontà del paziente» e che «il medico, se il paziente non è in grado di esprimere la propria volontà, deve tenere conto nelle proprie scelte di quanto precedentemente manifestato dallo stesso in modo certo e documentato». D'altra parte, in assenza di dichiarazioni anticipate di trattamento espresse dal paziente, la volontà dello stesso può esser ricostruita sulla base della personalità, dello stile di vita, delle inclinazioni, dei valori di riferimento e delle convenzioni etiche, religiose, culturali e filosofiche dell'interessato.

Ciò implica dunque che la scelta in merito ai trattamenti ai quali sottoporre o non sottoporre l'incapace debba essere compatibile «con la rappresentazione di sé sulla quale egli aveva costruito la sua vita fino a quel momento».

Secondo tali indicazioni, dunque, nell'ipotesi in cui non sia possibile l'applicazione del subjective standard, ovvero la considerazione delle indicazioni fornite in precedenza dal soggetto rappresentato, il tutore dovrebbe decidere sulla base del substituted judgement standard, che comporta la deduzione, sulla base della conoscenza che si ha del soggetto rappresentato, delle sue convinzioni religiose, dei valori etici di riferimento, delle aspettative di vita, della decisione che quest'ultimo avrebbe espresso se in grado di autodeterminarsi: tale criterio implicherebbe pertanto la formazione di un consenso "sostitutivo" attraverso la ricostruzione della presumibile volontà dell'interessato alla luce della storia personale dello stesso, e dunque la possibile realizzazione di una persona non più in grado di assumere decisioni in relazione al proprio stato di salute e alla propria vita [6,7]. 


\section{COMMENTO DEL CASO}

La situazione di incapacità delineata si ritrova nel caso di specie, relativo appunto ad una persona in stato vegetativo permanente ed in particolare alla richiesta avanzata dal tutore, di interruzione dei trattamenti di sostenimento vitale.

Secondo la Corte, partendo dal presupposto che «l'idratazione e l'alimentazione artificiali con sondino nasogastrico costituiscono un trattamento sanitario», e dunque la scelta relativa alla loro attivazione/sospensione è rimessa all'interessato ovvero al suo rappresentante, "al giudice non può essere richiesto di ordinare il distacco del sondino nasogastrico: una pretesa di tal fatta non è configurabile di fronte ad un trattamento sanitario, come quello di specie, che, in sé, non costituisce oggettivamente una forma di accanimento terapeutico, e che rappresenta, piuttosto, un presidio proporzionato rivolto al mantenimento del soffio vitale, salvo che, nell'imminenza della morte, l'organismo non sia più in grado di assimilare le sostanze fornite o che sopraggiunga uno stato di intolleranza, clinicamente rilevabile, collegato alla particolare forma di alimentazione».

Il giudice quindi in tal caso non può costituire il referente principale nel processo decisionale relativo al paziente incapace, dal momento che il nostro ordinamento individua nel rappresentante legale il soggetto legittimato ad esprimere il consenso ai trattamenti sanitari nell'interesse dell'incapace, sottoposto eventualmente ad un controllo da parte del giudice che potrebbe ad esempio - come nel caso di specie - decidere se autorizzare o meno la sospensione di un determinato trattamento sanitario richiesta dal tutore.

La Corte prende dunque anzitutto una posizione nel dibattito sullo stato vegetativo permanente riguardo alla natura di atto medico o di cura di base di nutrizione e idratazione artificiale, discostandosi nettamente dalla posizione recentemente espressa a maggioranza dal Comitato Nazionale per la Bioetica [8] secondo il quale «nella misura in cui l'organismo ne abbia un obiettivo beneficio, nutrizione ed idratazione artificiali costituiscono forme di assistenza di base ordinaria e proporzionata», ovvero "atti dovuti eticamente [...] in quanto indispensabili per garantire le condizioni fisiologiche di base per vivere».

In relazione al caso di specie, e ai criteri sopra individuati affinché il tutore nell'esercizio della sua attività di cura promuova effettivamente il miglior interesse dell'incapace, da determinarsi non solo in relazione alle componenti cliniche, tecniche ed oggettive, della situazione di specie, i giudici concludono che: "ove il malato giaccia da moltissimi anni (nella specie, oltre quindici) in stato vegetativo permanente, con conseguente radicale incapacità di rapportarsi al mondo esterno, e sia tenuto artificialmente in vita mediante un sondino nasogastrico che provvede alla sua nutrizione e idratazione, su richiesta del tutore che lo rappresenta, e nel contraddittorio con il curatore speciale, il giudice può autorizzare la disattivazione di tale presidio sanitario (fatta salva l'applicazione delle misure suggerite dalla scienza e dalla pratica medica nell'interesse del paziente), unicamente in presenza dei seguenti presupposti:

- quando la condizione di stato vegetativo sia, in base ad un rigoroso apprezzamento clinico, irreversibile e non vi sia alcun fondamento medico, secondo gli standard scientifici riconosciuti a livello internazionale, che lasci supporre la benché minima possibilità di un qualche, sia pure flebile, recupero della coscienza e di ritorno ad una percezione del mondo esterno;

- sempre che tale istanza sia realmente espressiva, in base ad elementi di prova chiari, univoci e convincenti, della voce del paziente medesimo, tratta dalle sue precedenti dichiarazioni ovvero dalla sua personalità, dal suo stile di vita e dai suoi convincimenti, corrispondendo al suo modo di concepire, prima di cadere in stato di incoscienza, l'idea stessa di dignità della persona.

Ove l'uno o l'altro presupposto non sussistano, il giudice deve negare l'autorizzazione, dovendo allora essere data incondizionata prevalenza al diritto alla vita, indipendentemente dal grado di salute, di autonomia e di incapacità di intendere $\mathrm{e}$ di volere del soggetto interessato e dalla percezione, che altri possano avere, della qualità della vita stessa».

La sentenza della Corte dunque, se da un lato rappresenta una «chiara riaffermazione che ogni atto medico trova la sua giustificazione nel consenso informato» anche nel caso di soggetti incapaci, attraverso il riconoscimento di un potere decisionale del tutto nuovo al rappresentante legale, d'altra parte attribuisce a quest'ultimo degli obblighi stringenti: al di fuori delle condizioni individuate (irreversibilità della condizione del paziente in stato vegetativo permanente e conformità della decisione alla volontà del paziente, sia essa espressamente trasmessa da quest'ultimo o ricostruita anche attraverso testimonianze o sulla base dello stile di vita e del sistema valoriale di riferimento dell'interessato), la sospensione della nutrizione e dell'idratazione artificiali «in nessun caso può essere basata su un giudizio di qualità (o di assenza di qualità) della vita formulato da persone diverse dal paziente, fossero pure lo stesso rappresentante legale o altri membri della famiglia» [9].

La decisione della Corte intende dunque affermare espressamente la centralità della volontà dell'interessato, anche incapace, concetto già evidenziato in relazione alla medesima condizione clinica oggetto del caso di specie nelle Conclusioni e 
Raccomandazioni della cosiddetta "Commissione Veronesi” ovvero il Gruppo di lavoro su nutrizione e idratazione nei soggetti in stato di irreversibile perdita della coscienza, istituito con Decreto del Ministro della sanità, del 20 ottobre 2000, che sanciva "la centralità della volontà del diretto interessato per ogni decisione che riguardi il proprio corpo, la propria salute e la propria vita» nonché «la riaffermazione di tale centralità anche per gli individui che si trovino in stato di irreversibile perdita di coscienza e la necessità pertanto di considerare valido criterio orientativo delle decisioni la volontà precedentemente espressa o ricostruibile attraverso testimonianze o in documenti, come direttive anticipate o living will o altre modalità similari».

La sentenza della Corte rappresenta dunque un importante contributo nel processo di riconoscimento delle manifestazioni anticipate di volontà; d'altra parte il riferimento non esclusivo a tali elementi nella ricostruzione della presumibile volontà dell'interessato non più in grado di autodeterminarsi, attraverso la considerazione di altre fonti utili a delineare il background culturale, etico, reli- gioso dello stesso, anche attraverso il ricorso a testimonianze, allontana dal rischio che una specifica regolamentazione delle dichiarazioni anticipate di trattamento, e dunque l'affidamento esclusivo alle indicazioni in esse contenute per la determinazione della scelta da compiere nel caso concreto, potrebbero comportare che altre forme di manifestazione di volontà non vengano ritenute efficaci, in quanto non esplicitamente previste dalla legge.

Si verificherebbe cioè il rischio che il principio di autonomia venga ad essere collegato unicamente a tali documenti, mentre "tale principio ha comunque valore indipendentemente dalla stesura di una direttiva» [10]; la Corte assume invece un'accezione ampia del principio di autonomia e del diritto all'autodeterminazione, sia dal punto di vista temporale (attraverso il riconoscimento di forme di manifestazione anticipate di volontà), sia dal punto di vista degli strumenti che possono concorrere nel far emergere la volontà del soggetto che anche in relazione ad un paziente incapace, assume dunque una posizione centrale di preminenza nel processo del decision making.

\section{BIBLIOGRAFIA}

1. Comitato Nazionale per la Bioetica. Informazione e consenso all'atto medico, Presidenza del Consiglio dei Ministri - Dipartimento per l'Informazione e l'Editoria, 20 giugno 1992, 30

2. Ferrando G. I diritti della persona e la sospensione dei trattamenti medici. Bioetica. Rivista Interdisciplinare 2005; 2: 158

3. Santosuosso A. Novità e remore sullo "stato vegetativo persistente". Foro It 2000: 2032

4. Morelli MC. Un passo avanti per l'uguaglianza tra competenti e incompetenti. Bioetica. Rivista Interdisciplinare 2001; 2: 280

5. Ferrando G. I diritti della persona e la sospensione dei trattamenti medici. Bioetica. Rivista Interdisciplinare 2005; 2: 163

6. Yarborough M. Adults are not big children: examining surrogate consent to research using adults with dementia. Cambridge Quarterly of Healthcare Ethics 2002; 2: 160-8

7. Guinn DE. Mental competence, caregivers, and the process of consent: research involving Alzheimer's patients or others with decreasing mental capacity. Cambridge Quarterly of Healthcare Ethics 2002; 3: 238

8. Comitato Nazionale per la Bioetica. Lalimentazione e l'idratazione di pazienti in stato vegetativo persistente, 30 settembre 2005

9. Defanti CA. Eluana Englaro: la fine di un incubo? Bioetica. Rivista interdisciplinare 2007; 4: 111-2

10. Bailo R, Cecchi P. Direttive anticipate e diritto a rifiutare le cure: aspetti etici e giuridici. Rass Dir Civ 1998; 3: 496

\section{CORRESPONDING AUTHOR}

Dott.ssa Paola Delbon, Cattedra di Medicina Legale, P.le Spedali Civili, 1 - 25123 Brescia 